\title{
Enhanced UnaG With Minimal Labeling Artifact for Single-Molecule Localization Microscopy
}

\author{
Sangyoon Ko ${ }^{1,2 \dagger}$, Jiwoong Kwon ${ }^{1 * t \neq}$ and Sang-Hee Shim ${ }^{1,2 *}$ \\ ${ }^{1}$ Center for Molecular Spectroscopy and Dynamics, Institute of Basic Science, Seoul, South Korea, ${ }^{2}$ Department \\ of Chemistry, Korea University, South Korea
}

OPEN ACCESS

Edited by:

Periklis Pantazis,

Imperial College London,

United Kingdom

Reviewed by:

Takeharu Nagai,

Osaka University, Japan

Kyra J. E. Borgman,

Institut Curie, France

*Correspondence:

Jiwoong Kwon

jkwon34@jh.edu

Sang-Hee Shim

sangheeshim@korea.ac.kr

$\ddagger$ Present address:

Jiwoong Kwon,

Department of Biophysics and Biophysical Chemistry, School

of Medicine, Johns Hopkins

University, Baltimore, MD,

United States

${ }^{t}$ These authors have contributed equally to this work and share first authorship

Specialty section:

This article was submitted to Biophysics,

a section of the journal

Frontiers in Molecular Biosciences

Received: 30 December 2020 Accepted: 23 March 2021

Published: 20 April 2021

Citation:

Ko S, Kwon J and Shim S-H (2021) Enhanced UnaG With Minimal Labeling Artifact for Single-Molecule

Localization Microscopy.

Front. Mol. Biosci. 8:647590. doi: 10.3389/fmolb.2021.647590
We introduced enhanced UnaG (eUnaG), a ligand-activatable fluorescent protein, for conventional and super-resolution imaging of subcellular structures in the mammalian cells. eUnaG is a V2L mutant of UnaG with twice brighter bulk fluorescence. We previously discovered the reversible fluorescence switching behavior of UnaG and demonstrated the high photon outputs and high localization numbers in single-molecule localization microscopy (SMLM). In this study, we showed that the fluorescence of eUnaG can be switched off under blue-light illumination, while a high concentration of fluorogenic ligands in the buffer can efficiently restore the fluorescence, as in UnaG. We demonstrated the capacity of eUnaG as an efficient protein label in mammalian cells, as well as for SMLM by utilizing its photoswitchable nature. While cytosolic UnaG proteins showed aggregated patches and fluorescence reduction at high expression levels, eUnaG-labeled protein targets successfully formed their proper structures in mammalian cells without notable distortion from the endogenous structure in the majority of transiently expressing cells. In particular, eUnaG preserved the vimentin filament structures much better than the UnaG. eUnaG provided similarly high singlemolecule photon count distribution to UnaG, thus also similarly high resolution in the super-resolution images of various subcellular structures. The sampling coverage analysis of vimentin filaments in SMLM images showed the improvement of labeling efficiency of eUnaG. eUnaG is a high-performance fluorescent protein for fluorescence and single-molecule localization imaging in green emission with minimal labeling artifact.

Keywords: fluorescent protein, UnaG, photoswitching, super-resolution (SR) imaging, bilirubin, single-molecule localization microscopy

\section{INTRODUCTION}

Fluorescent proteins (FPs) are workhorses in live-cell fluorescence microscopy due to the facile and specific labeling of the target proteins (Chudakov et al., 2010; Rodriguez et al., 2017). Likewise, FPs have been extensively used in single-molecule localization microscopy (SMLM) (Zhou and Lin, 2013; Shcherbakova et al., 2014; Sauer and Heilemann, 2017). The first demonstrations of SMLM used photoactivatable fluorescence proteins (Betzig et al., 2006; Hess et al., 2006) along with organic dyes (Rust et al., 2006; Sharonov and Hochstrasser, 2006). The on-off transition of fluorescence emission is required in the SMLM for the temporal separation of individual molecules within the diffraction-limited area, allowing high precision localization of the individual molecules. The quality of the resultant super-resolution image is determined by two photophysical characteristics 
of the fluorophores. The photon number emitted from the fluorescent state determines the localization precision of determining the centroid position of a single fluorophore (Thompson et al., 2002). The number of switching cycles and the fraction of time spent in the fluorescent state, termed as the onoff duty cycle, are related to the labeling density and the Nyquist resolution (Shroff et al., 2008). Most of the FPs offer lower photon numbers than the organic fluorophores, hence resulting in lower spatial resolutions (Patterson and Lippincott-Schwartz, 2002; Subach et al., 2009; Brakemann et al., 2011). Furthermore, FPs often show irreversible fluorescence transition that restricts the spot density or transition between two different emission states that complicates multicolor applications (McKinney et al., 2009).

Recently, we introduced UnaG protein as an efficient SMLM probe for multicolor live-cell imaging (Kwon et al., 2020). UnaG is a ligand-activatable FP with a fluorogenic ligand, bilirubin (Kumagai et al., 2013). The ligand and apoUnaG protein are non-fluorescent in solution and become fluorescent upon binding to form holoUnaG. Photoswitching of UnaG is mediated by repetitive binding of bilirubin after photooxidation of the ligand followed by detachment of the damaged bilirubin from the protein. UnaG offers the highest photon numbers among blue-absorbing FPs and easily controllable switching kinetics. The off-switching rate is controlled by excitation intensity and oxygen concentration. The on-switching rate is linear to the concentration of bilirubin, whose fluorescence is undetectable up to micromolar concentration for supporting the repetitive recovery of fluorescence.

Here we investigated the photoswitching nature of enhanced UnaG (eUnaG) protein and its application to SMLM. eUnaG is a single-mutated variant of $\mathrm{UnaG}$, in which Val 2 is substituted with leucine (Yeh et al., 2017). The single mutation near the $\mathrm{N}$-terminus boosts the brightness of bacterial expression to about twofolds (Yeh et al., 2017). Since UnaG is a suitable fluorescent protein for SMLM, we measured the single-molecule photophysical characteristics of eUnaG and explored the SMLM imaging capability. We found that lower aggregation tendency of eUnaG leads to the benefit in the morphology and brightness of cellular structures, especially in the case of vimentin filament, whose morphology is sensitive to the fused tags.

\section{MATERIALS AND METHODS}

\section{Plasmids}

Genetic incorporation using standard restriction enzymes and T4 DNA ligase was performed to construct eUnaG plasmids. eUnaG mutation (V2L) was performed via QuikChange sitedirected mutagenesis protocol on the UnaG-mCherry construct. eUnaG-mCherry construct was utilized for PCR template for other eUnaG constructs. The primers for PCR amplification contain the sequences for small epitope tags such as HA or Flag. PCR products were digested with restriction enzymes and ligated into cut vectors (e.g., pcDNA3 and pDisplay). For the expression in mammalian cells, the CMV promoter was introduced in all cases. More detailed information about the genetic constructs is summarized in Supplementary Table 1.

\section{Cell Culture, Transfection, and Fixation}

Cos-7 cells [Korean Collection for Type Cultures (KCTC)] were grown in Dulbecco's modified Eagle's medium (DMEM, SH30022.01, Hyclone, UT, United States) containing 10\% v/v fetal bovine serum (97068-085, VWR Life Science, PA, United States) and 1\% v/v antibiotic-antimycotic (15240-062, Gibco, MD, United States). The cells were electroporated (MPK5000, Invitrogen, CA, United States) during the general subculture, with $\sim 500$ ng of proper plasmid, and seeded on a coverslipbottomed 8-well chamber (155409, Lab-Tek, MI, United States) at the density of $5 \times 10^{4}$ per well. After $24-48 \mathrm{~h}$ of transfection, the cells were fixed in 3\% paraformaldehyde (50980-495, Electron Microscopy Sciences, PA, United States) with 0.2\% glutaraldehyde (16020, Electron Microscopy Sciences, PA, United States) for $10 \mathrm{~min}$ at room temperature.

\section{Imaging Buffers}

Photon statistics and super-resolution imaging of eUnaG were performed in imaging buffer based on Tris $\mathrm{pH} 8$ (10 mM, TRI01, LPS Solution, Daejeon, South Korea) containing $\mathrm{NaCl}(50 \mathrm{mM}$, 7548-4405, Daejung, Siheung, South Korea) and $\beta$-D-glucose (10\% v/v, G7021, Sigma, MD, United States). To reduce the concentration of dissolved oxygen, we added an enzyme-based oxygen scavenging system (GLOX) containing glucose oxidase (560 $\mu \mathrm{g} / \mathrm{ml}, \mathrm{G} 2133$, Sigma, MD, United States) and catalase (400 $\mu \mathrm{g} / \mathrm{ml}, \mathrm{C} 100$, Sigma, MD, United States); $1 \mu \mathrm{M}$ of external bilirubin (B4126, Sigma, MD, United States) was supplied to the imaging buffer during the SMLM imaging.

\section{Fluorescence Microscope}

A commercially available spinning-disk confocal system (DragonFly, Andor, Belfast, United Kingdom) was used to acquire the confocal images of vimentin filament to compare the expression pattern (Figures 1F,H). A confocal-based multi-acquisition system, TCS SP8 from Leica with a TCSPC (HydraHarp 400, PicoQuant, Berlin, Germany), was used to measure the fluorescence lifetimes. Photon statistics, widefield, and SMLM images were obtained using a homebuilt imaging system; 405-, 488-, 561-, and 647-nm laser light sources (405 nm: $200 \mathrm{~mW}$, OBIS, Coherent, CA, United States; $488 \mathrm{~nm}$ : $150 \mathrm{~mW}$, OBIS, Coherent, CA, United States; $561 \mathrm{~nm}$ : $150 \mathrm{~mW}$, OBIS, Coherent, CA, United States; $647 \mathrm{~nm}$ : $120 \mathrm{~mW}$, OBIS, Coherent, CA, United States) coupled with a single-mode optical fiber (3.4 $\mu \mathrm{m}$ diameter, PM-488-FC, Thorlabs, NJ, United States) were collimated, expanded about 25 times, and delivered into a microscope (Eclipse Ti-E, Nikon, Tokyo, Japan) through a lens $(f=400 \mathrm{~mm}$, AC508200-A-ML, Thorlabs, NJ, United States) and a dichroic mirror (ZT405/488/561/647rpc, Chroma, VT, United States) to make widefield illumination on the sample plane in epi geometry. The fluorescence was collected by an objective lens (Plan Apo TIRF, $100 \times$, NA 1.49, oil, Nikon, Tokyo, Japan) and filtered by an emission filter (ZET405/488/561/647m-TRF, Chroma, VT, United States). A focus maintaining system embedded in the microscope precisely kept the focal plane during the measurement. A back-illuminated scientific CMOS camera 
A
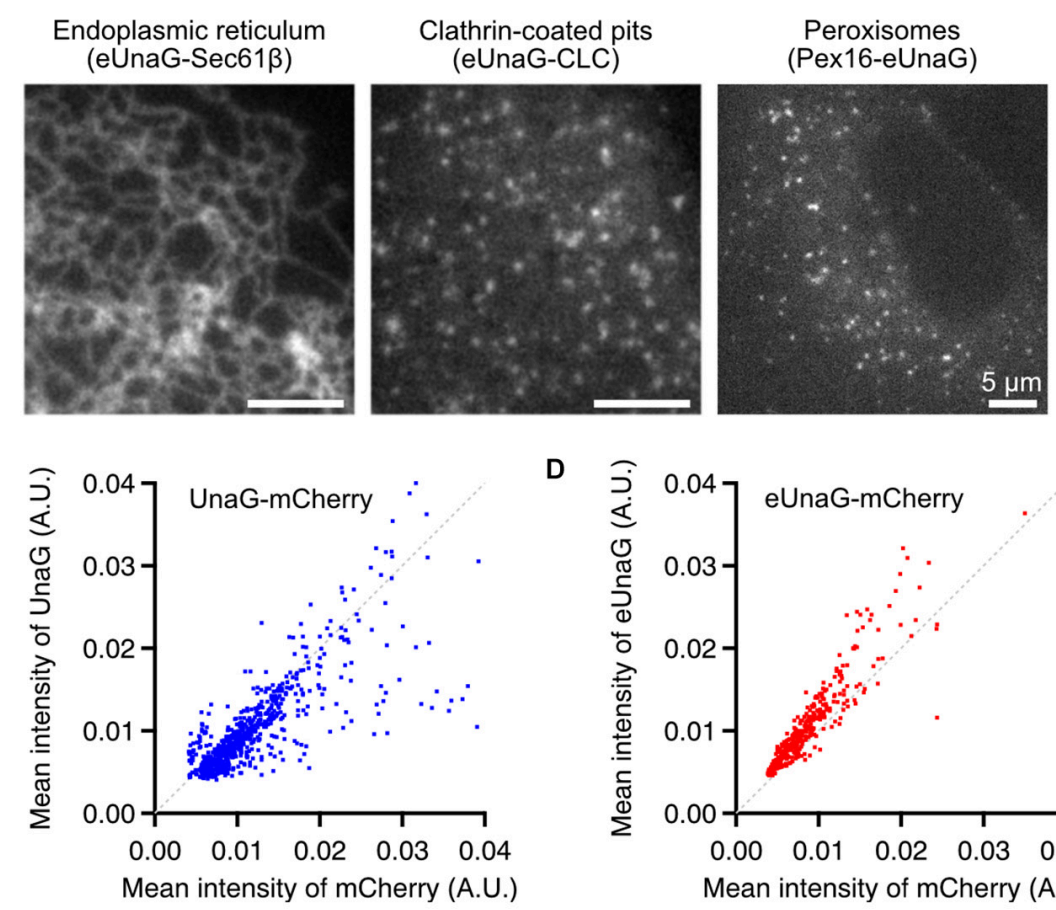

$\mathbf{F}$
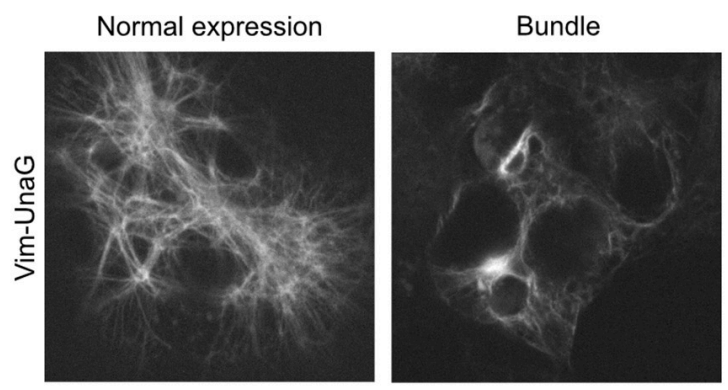

H

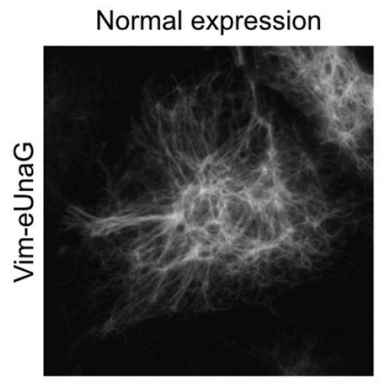

D
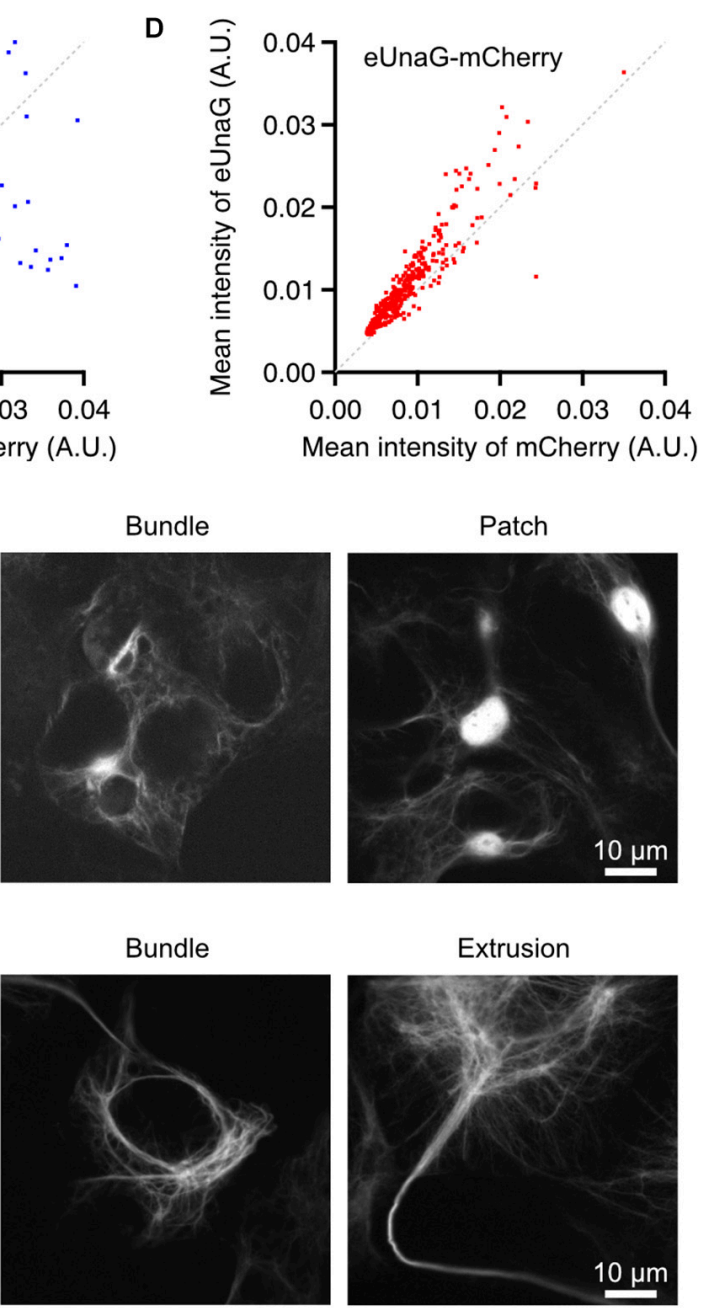

B

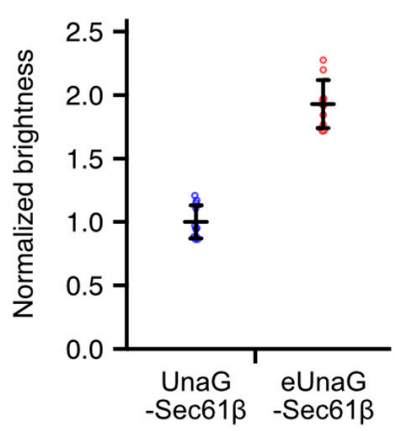

$\mathbf{E}$

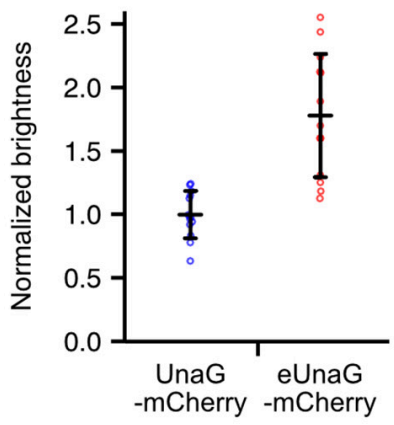

G

UnaG-labeled vimentin filaments (24 h post-transfection)

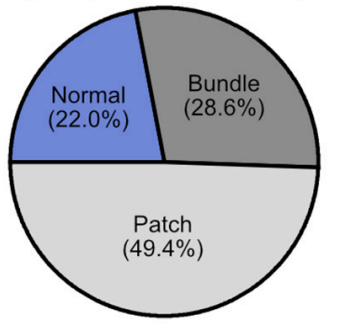

I eUnaG-labeled vimentin filaments ( $24 \mathrm{~h}$ post-transfection)

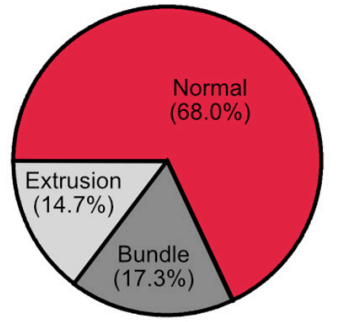

FIGURE 1 | Mammalian expression of eUnaG-labeled protein in Cos-7 cells. (A) Widefield images of eUnaG-labeled Sec61 $\beta$, CLC, and Pex16 proteins for visualization of the endoplasmic reticulum, clathrin-coated pits, and peroxisomes, respectively. (B) Fold increase in the fluorescence intensities of eUnaG [red dots, $n=10$ fields of view (FOVs)] compared to those of UnaG (blue dots, $n=11$ FOVs) measured from UnaG-Sec61 $\beta$ fusion proteins. Each data point is the mean intensity of the entire FOV of a confocal fluorescence image containing tens of cells. (C,D) Fluorescence intensity distribution at various expression levels of cytosolic UnaG-mCherry (C) and eUnaG-mCherry (D) fusion proteins. Each data point was measured from a cell detected from a large-FOV confocal image by CellProfiler ( $n=803$ for UnaG, $n=459$ for eUnaG). The gray dotted line is drawn along $y=x$ in (C,D). (E) Fold increase in the fluorescence intensities of eUnaG (red dots, $n=13$ FOVs) from those of UnaG (blue dots, $n=13 \mathrm{FOV}$ ) measured from confocal images of Cos7 cells expressing cytosolic UnaG-mCherry proteins. The fluorescence intensities from UnaG and eUnaG proteins were normalized to the mCherry fluorescence intensities. Each data point is the mean intensity of the entire FOV containing tens of cells. (F) Confocal images of the expression pattern of UnaG-labeled vimentin filaments in Cos-7 cells. The cells were fixed $24 \mathrm{~h}$ after the transfection. (G) Phenotype distribution of cells expressing Vim-UnaG quantified from (F) $(n=91$ cells). (H) Example confocal images of the expression pattern of eUnaG-labeled vimentin filaments. The cells were fixed $24 \mathrm{~h}$ after the transfection as in (F). (I) Phenotype distribution of the expression pattern of Vim-eUnaG proteins obtained from $\mathbf{( H )}(n=75$ cells). Error bars: SDs. 
(sCMOS, Prime95b, Photometrics, AZ, United States) collected the filtered fluorescence signal through the tube lens, resulting in an image pixel size of $110 \mathrm{~nm}$. The acquisition parameters were set to 12-bit sensitive detection mode with no pre- and post-processing.

\section{Data Acquisition and Analysis Software}

Fusion, a commercial acquisition software from Andor, was used to acquire confocal images from the DragonFly confocal microscope. SymPhoTime64 from PicoQuant was used to acquire and analyze the fluorescence lifetime. To measure the intensities of UnaG- and eUnaG-fused Sec61 $\beta$ and mCherry, several confocal images containing tens of cells were obtained and analyzed by CellProfiler, an open-source software for cell image processing. An open-source imaging software, $\mu$ Manager, was used to control the sCMOS camera and to acquire the singlemolecule images (Edelstein et al., 2014). The raw single-molecule movies were processed by a high-density analysis ImageJ plugin, HAWK, to disentangle overactivated and overlapped fluorescence spots (Marsh et al., 2018). The processed singlemolecule movies were analyzed by ThunderSTORM, an ImageJ plugin, to localize the centroid positions and reconstruct SMLM images (Ovesný et al., 2014). Sampling coverages of vimentin filaments were analyzed using the basic functions of ImageJ and a home-build MATLAB code, by referring to a previous report (Virant et al., 2018). We used the term "sampling coverage" instead of "labeling coverage" used in the study by Virant et al. (2018), which can give a false impression that it is calculated from the number of labeled copies and endogenous non-fluorescent copies of vimentin. Following the procedures mentioned in the study by Virant et al. (2018), from SMLM images, individual vimentin filaments were manually selected and straightened using ImageJ. The home-built MATLAB code binarized the result images and calculated the fraction of the covered area of the middle three pixels to calculate the sampling coverage.

\section{RESULTS}

\section{Enhanced UnaG Expression in a Mammalian Cell}

Although UnaG protein has been used for imaging applications of mammalian cells such as local bilirubin quantification (Park et al., 2016), biomolecular interactions (To et al., 2016), and super-resolution visualization of subcellular structures (Kwon et al., 2020), there is no report that uses eUnaG in mammalian cells to the best of our knowledge. To demonstrate its potential to visualize the subcellular structures in mammalian cells, we fused eUnaG with various proteins such as Sec61 $\beta$, Vim, clathrin light chain (CLC), Pex16, and cytosolic mCherry; transiently expressed the fusion proteins in Cos-7 cells; and observed the expression patterns (Figure 1A and Supplementary Figure 1). All the eUnaG-labeled proteins formed their respective subcellular structures, without notable distortion in their structures for the majority of cells. When we directly compare the fluorescence intensity from UnaG- and eUnaG-labeled Sec61 $\beta$ proteins, eUnaG showed $\sim 2$ times brighter signals (Figure 1B).
To normalize the fluorescence intensities by the expression levels varying in transiently expressing cells, we expressed the cytosolic mCherry fused with UnaG or eUnaG. At the level of individual cells, the UnaG intensity was largely proportional to the mCherry intensity (Figure 1C). However, a noticeable portion of cells expressing UnaG-mCherry showed deviation from the linear dependence when the mCherry intensity was high (Figure 1C). In contrast, the eUnaG intensity was mostly linear to the mCherry intensities with few exceptions (Figure 1D). Also, the normalized intensities of eUnaG to mCherry appear to be slightly higher than those of the linear fraction of UnaG. Assuming that the mCherry intensity is proportional to the concentration of the fusion protein molecules in each transiently expressing cell, these results can be interpreted as the concentration-dependence of the fluorescence intensities of UnaG and a slight increase of eUnaG fluorescence. The high concentration of cytosolic UnaG could induce UnaGmediated protein aggregation, resulting in loss of fluorescence via aggregation-induced quenching or inhibition of ligand binding. Since the fluorescence lifetimes of $\mathrm{UnaG}$ and eUnaG are very similar under the same measurement condition (Supplementary Figure 2), this aggregation-induced reduction of UnaG fluorescence could contribute to the bulk brightness difference between UnaG and eUnaG. The ensemble fluorescence intensities of UnaG and eUnaG from cytosolic UnaG- and eUnaG-mCherry fusion proteins showed approximately twofold enhancement of eUnaG fluorescence, when normalized by mCherry signal (Figure 1E). This is consistent with the result from Sec61 $\beta$ fusion proteins (Figure 1B) and the previous result from bacterial expression (Yeh et al., 2017).

To compare the degree of aggregation, we examined the induced formation of stacked ER membrane whorls, termed OSER (organized smooth ER), which are commonly used for the characterization of the oligomerization levels of FPs (Costantini et al., 2012). We compared UnaG-Sec61b and eUnaG-Sec61b OSERs to mMaple3-Sec61b OSERs (Supplementary Figure 3). Previously, mMaple3 was classified as monomeric by counting OSER in CytERM-mMaple3 fusions (Kaberniuk et al., 2018). In our Sec61b-based assays, UnaG and eUnaG showed a lower level of oligomerization than mMaple3 (Supplementary Figure 3), indicating the monomeric character of UnaG and eUnaG. Also, eUnaG showed a slightly lower level of aggregation than that of UnaG, supporting the possibility of aggregation-induced reduction of fluorescence.

We further examined the expression pattern of vimentin filaments labeled with UnaG and eUnaG. Vimentin filaments have been used as a reference structure for evaluating labeling artifacts because the cellular structure is sensitive to the multimeric character of the fused FPs (Wang et al., 2014). Most of the FPs for SMLM are mutants of multimeric FPs, and the residual tendency to multimerize can induce abnormally thick fiber bundles of vimentin filament (Wang et al., 2014). For instance, mMaple3, considered as monomeric by OSER (Kaberniuk et al., 2018), showed $\sim 30 \%$ of cells with normal vimentin filaments and $\sim 70 \%$ of cells with abnormal phenotypes (Supplementary Figure 4). When we transiently expressed UnaG-labeled vimentin for $24 \mathrm{~h}$, only $\sim 20 \%$ of fluorescent 
cells showed normal vimentin structures of thin filaments (Figures 1F,G and Supplementary Figure 5), and the remaining cells showed abnormal expression patterns such as bundled filaments ("Bundle" in Figure 1F) and bright fluorescent patches ("Patch" in Figure 1F). The "Patch" structures are consistent with the non-linear behavior of UnaG intensities at high expression level (Figure 1C). These abnormal structures may stem from the aggregation of Vim-UnaG proteins in high cytosolic concentrations. When observing the phenotypes after 4 days of electroporation, $\sim 65 \%$ of UnaG-expressing cells showed normal vimentin distribution (Supplementary Figure 6), indicating that low expression levels help to prevent aggregation from cytosolic UnaG, while the Vim-UnaG proteins incorporated into the filaments did not perturb the filamentous structure. In contrast, $\sim 70 \%$ of Vim-eUnaG expressing cells showed a normal expression pattern of thin vimentin filaments after $24 \mathrm{~h}$ from the transfection (Figures $\mathbf{1 H}, \mathbf{I}$ and Supplementary Figure 5); $\sim 30 \%$ of Vim-eUnaG-expressing cells showed long, thick bundle of vimentin fibers as the major artifact. These bundled filaments were formed in either cytosolic area around the nucleus ("Bundle" in Figure $\mathbf{1 H}$ ) or cell periphery forming extrusions reaching out of the cell ("Extrusion" in Figure 1H). Interestingly, Vim-eUnaG-expressing cells did not show "Patch" phenotype found in $\sim 50 \%$ of Vim-UnaG-expressing cells. This is consistent with the OSER results, indicating the lower aggregation tendency of eUnaG (Supplementary Figure 3). Thus, eUnaG can serve as a facile fluorescent marker for artifact-free imaging applications to visualize various subcellular structures in the mammalian cells by protein fusion and transient expression, without any severe distortions in the target structures for the majority of cells.

\section{Photoswitching Nature of eUnaG}

Since eUnaG is a single-residue mutant of UnaG at the $\mathrm{N}$-terminus, we postulated that the fluorescence of eUnaG can be switched off by light illumination and turn back on by rebinding with the fluorogenic ligand, bilirubin, like UnaG. We tested the photoswitching behavior of eUnaG with eUnaGSec61 $\beta$ expressing Cos-7 cells (Figure 2A). Upon a high-intensity illumination of $488-\mathrm{nm}$ laser light $\left(300 \mathrm{~W} / \mathrm{cm}^{2}\right)$, the fluorescence from eUnaG quickly bleached off within $10 \mathrm{~s}$. When we treated the bleached eUnaG proteins with $1 \mu \mathrm{M}$ of exogeneous bilirubin, the fluorescence recovered in high efficiency, allowing the structure of the endoplasmic reticulum to be clearly visualized again. The on-off switching cycle could be repeated multiple times as observed in UnaG, and the recovery efficiency was quite similar to UnaG during the repetitive switching cycles (Figures 2A,B).

This common way of reversible switching indicates that the photoswitching mechanism of eUnaG is similar to that of UnaG. Indeed, when we measured the off-switching rate in different concentrations of dissolved oxygen, there were two different offswitching speeds $\left(\mathrm{k}_{\mathrm{off} 1}\right.$ and $\left.\mathrm{k}_{\mathrm{off} 2}\right)$ as in $\mathrm{UnaG}$, corresponding to the two fluorescent forms of UnaG: less bright conformation that forms immediately after binding to bilirubin, known as UnaG1; brighter conformation (UnaG2) that spontaneously converts from UnaG1 (Figure 2C; Shitashima et al., 2017; Kwon et al., 2020). Both the off-switching rates of $\mathrm{k}_{\mathrm{off} 1}$ and $\mathrm{k}_{\mathrm{off} 2}$ for eUnaG were highly dependent on the concentration of dissolved oxygen, implying the similarity of off-switching mechanism that is mediated by the photo-oxidation of bilirubin molecule under intense 488-nm illumination (Figure 2D).

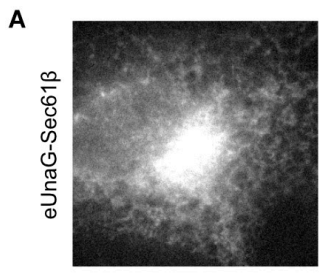

B

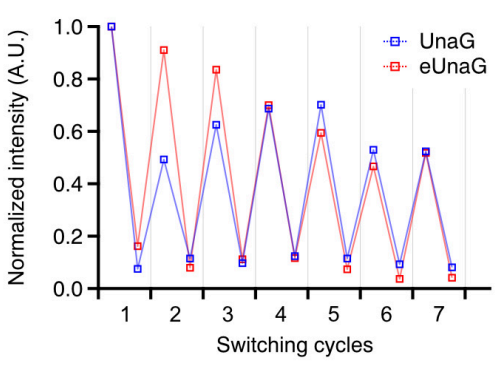

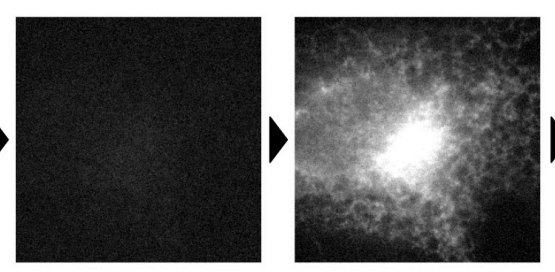

C

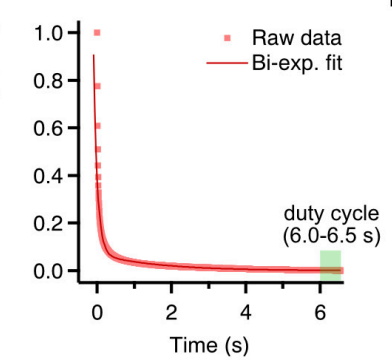$$
\text { D }
$$

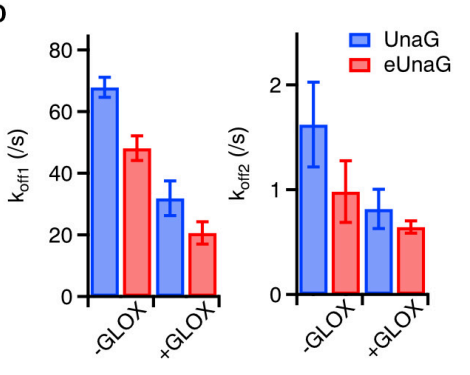

FIGURE 2 | Photoswitching behavior of eUnaG. (A) Widefield images of a fixed eUnaG-Sec61 $\beta$ expressing Cos-7 cell showing repetitive fluorescence switching of eUnaG. Images were obtained from two successful rounds of fluorescence switching (off-switching: $300 \mathrm{~W} / \mathrm{cm}^{2}$ of 488 -nm laser for 20 s; on-switching: $1 \mu \mathrm{M}$ bilirubin for $5 \mathrm{~min}$ ). (B) Repetitive on- and off-switching of UnaG and eUnaG proteins. Fluorescence intensities were measured from a series of widefield images as in (A) and normalized to the initial value. (C) Fluorescence intensity trace of eUnaG under $300 \mathrm{~W} / \mathrm{cm}^{2}$ of 488 -nm laser illumination. The decaying profile was well fitted by a bi-exponential function, giving two off-switching rates, $\mathrm{k}_{\text {off1 }}$ and $\mathrm{k}_{\mathrm{off} 2}$. The duty cycle was calculated from the average intensity at the equilibrium state (6.0-6.5 s). (D) Two off-switching rates of UnaG and eUnaG under different concentrations of dissolved oxygen (number of measurements: 5 for UnaG -GLOX, 14 for UnaG +GLOX, 6 for eUnaG -GLOX, 12 for eUnaG +GLOX). UnaG and eUnaG showed a similar tendency of slower off-switching rates with lower concentration of dissolved oxygen (+GLOX), in both $\mathrm{k}_{\text {off } 1}$ and $\mathrm{k}_{\mathrm{off} 2}$. Error bars: SDs. 
From the fluorescence intensity trace under off-switching condition, we can measure the on-off duty cycle from the ratio of the equilibrium intensity to the initial, unbleached intensity (Dempsey et al., 2011). With $300 \mathrm{~W} / \mathrm{cm}^{2}$ of illumination intensity, the duty cycle of eUnaG was measured as 0.0015 within $\sim 7 \mathrm{~s}$, which is comparable to the best-performing SMLM dyes such as Alexa 647 (Figure 2C).

The number of photons during the on-time of each switching cycle determines the localization accuracy in SMLM (Thompson et al., 2002). The photon number per switching cycle of eUnaG was saturated at a slightly higher level than that of UnaG as the exposure time increased (Figure 3A, saturation level: 1,270 photons for UnaG; 1,390 photons for eUnaG). The slight increase in the photon numbers of the eUnaG is consistent with the slight increase in the slope of the eUnaG/mCherry intensity plot (Figures 1C,D) and the slight decrease in the off-switching rate (Figure 2D). The average photon number value was 1,061 counts with $200 \mathrm{~ms}$ of exposure time, corresponding to the theoretical localization accuracy of $\sim 22 \mathrm{~nm}$ (Figure 3B). We measured the localization accuracy by the nearest neighbor analysis and obtained $15.7 \mathrm{~nm}$ for eUnaG, which is similar to the value obtained for UnaG (Figure 3C; Kwon et al., 2020). Note that the photon number of UnaG was on par with mMaple3, one of the brightest fluorescent proteins for SMLM (Wang et al., 2014; Kwon et al., 2020). With the even higher photon count of eUnaG, we can expect further resolution enhancement in SMLM images.

\section{Single-Molecule Localization Microscopy of eUnaG-Labeled Subcellular Structures}

As in UnaG, we obtained SMLM images of Sec61 $\beta$, Vim, and CLC labeled with eUnaG (Figure 4). These images were obtained with $1 \mu \mathrm{M}$ exogeneous bilirubin under widefield Epi illumination. In this condition, the fluorescence background was nearly unchanged since bilirubin is non-fluorescent in solution and becomes fluorescent only when bound to the protein (i.e., bilirubin is fluorogenic) (Kwon et al., 2020). Also, the on-switching rates and the accumulated localization number per SMLM image could be controlled by the bilirubin concentration. The combination of high photon number and high localization number from reversible, controllable switching of eUnaG allowed us to obtain high SMLM resolution that is determined by both localization precision (determined by photon number) and Nyquist resolution (determined by localization density) (Thompson et al., 2002; Shroff et al., 2008). The raw single-molecule movies were obtained for $>16,000$ frames with $20 \mathrm{~ms}$ of exposure time and were analyzed by HAWK software to reduce the unwanted artifacts from overlapping single-molecule images (Supplementary Figure 7; Marsh et al., 2018). The side-by-side comparison of widefield and SMLM images showed the resolution enhancement by SMLM imaging, and the beneficial photophysical characteristics of eUnaG enabled clear visualization of the ring-like structure of clathrin-coated pits (Figure 4B). Also, the average width of vimentin filaments measured by the full width in half maximum (FWHM) was $\sim 57 \mathrm{~nm}$ (Supplementary Figure 8). The widths of vimentin filaments imaged with UnaG and eUnaG were broader than those with Alexa 647, a standard best-performing organic dye for SMLM, but narrower than those with mMaple3, one of the SMLM FPs with the highest photon numbers (Supplementary Figure 9). Since Alexa 647 was linked to vimentin through streptavidin and AviTag (a peptide tag composed of 12 amino acids), the linkage error (i.e., the distance between the target protein and the fluorophore) can be approximated as roughly the same with FP-fused fibrils. Regarding the $\sim 10-\mathrm{nm}$ width of vimentin fibrils, the measured widths from SMLM images were consistent with the localization accuracies measured from the nearest neighbor analysis (NeNA) of vimentin SMLM images (Supplementary Figure 9), except for mMaple3 with low fiber sampling coverage (Endesfelder et al., 2014; Kwon et al., 2020).

Even though the fractions of cells with normal expression patterns differ dramatically for UnaG and eUnaG (Figures 1F-I), we could still find cells with normal vimentin structures and obtain SMLM images. From the SMLM images of vimentin fibers, we quantified the fiber sampling coverage calculated from the longitudinal fraction of fibers sampled by the localization points (Figures 4C-F and Supplementary Figure 10;

\section{A}

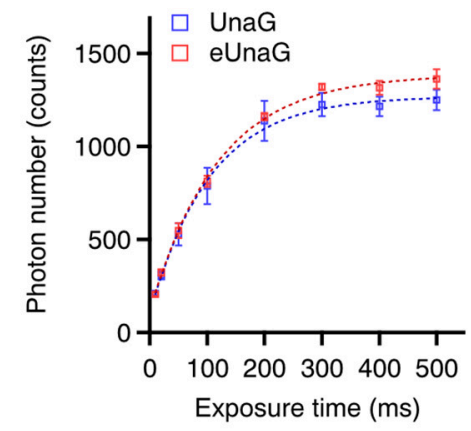

B

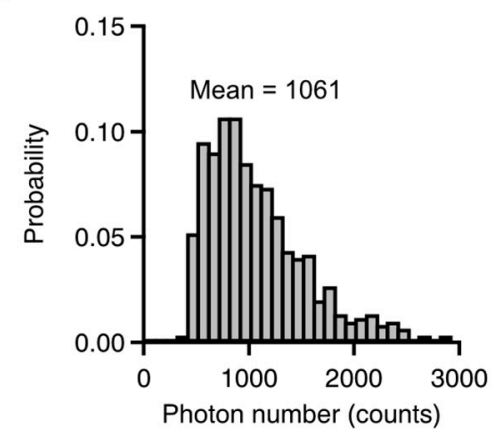

C

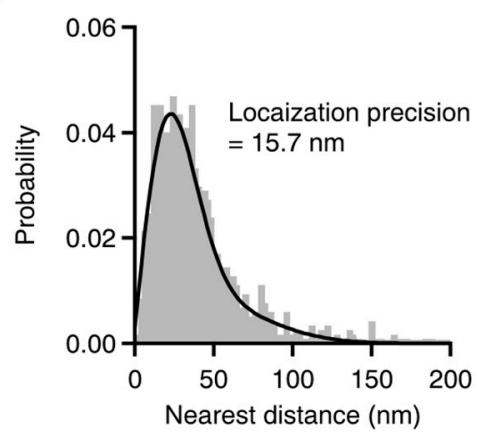

FIGURE 3 | Single-molecule switching characteristics of eUnaG. (A) Measured photon counts with different exposure times ( $n=8$ independent measurements). The saturation levels of photon counts were 1,270 and 1,390 for UnaG and eUnaG, respectively, indicating a slightly higher photon number of eUnaG. (B) Photon number distribution of eUnaG with $200 \mathrm{~ms}$ of exposure time, showing 1,061 mean photon counts corresponding $\sim 22 \mathrm{~nm}$ of theoretical spatial resolution. (C) Resolution measurement by the nearest neighbor analysis yielded $\sim 16 \mathrm{~nm}$ of spatial resolution from repetitive localization of eUnaG. Error bars: SDs. 


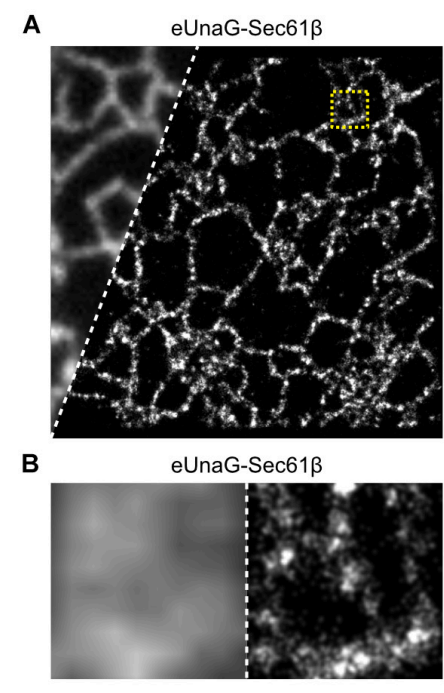

C

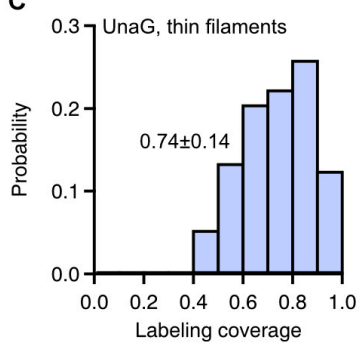

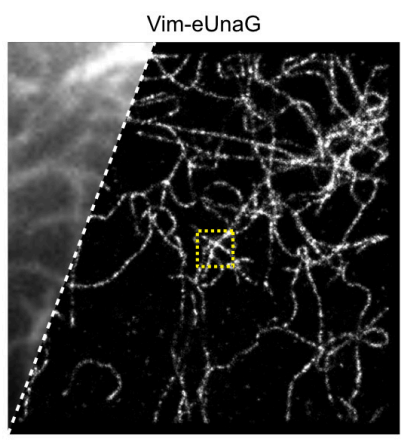

Vim-eUnaG
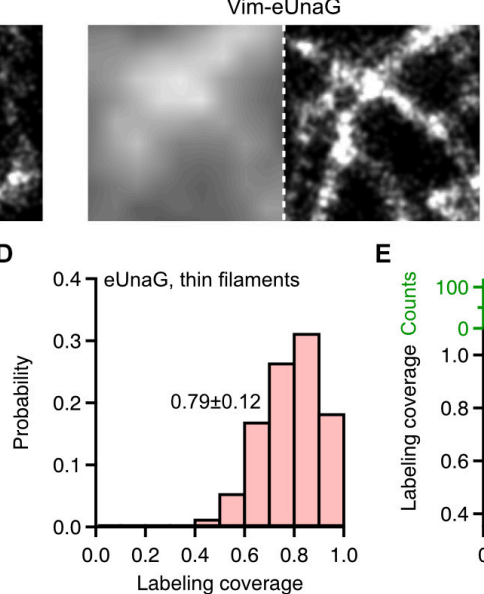

E

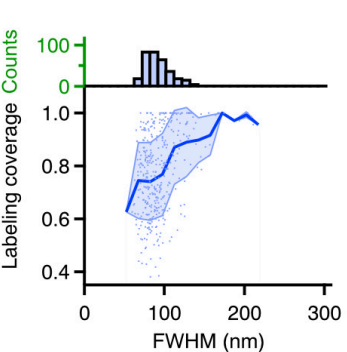

eUnaG-CLC

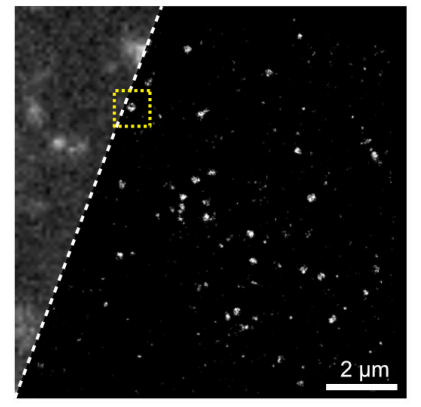

eUnaG-CLC
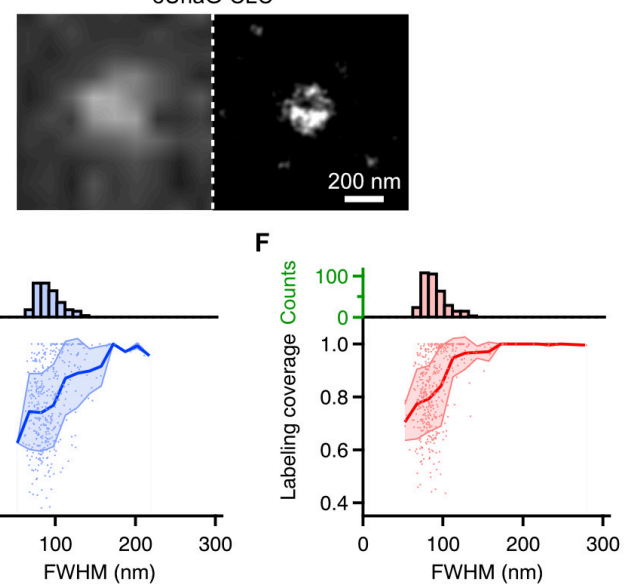

FIGURE 4 | Single-molecule localization microscopy of eUnaG-labeled subcellular structures in fixed Cos-7 cells. (A) Widefield (left) and SMLM (right) images of Cos-7 cells transfected with eUnaG-Sec61ß, Vim-eUnaG, and eUnaG-CLC. (B) Zoom-in images from yellow-dotted boxes in (A). Each panel contains widefield (left) and SMLM (right) images. (C,D) Sampling coverage of thin vimentin filaments fused with (C) UnaG and (D) eUnaG, obtained from corresponding SMLM images. $n=102$ and 180 filaments for UnaG and eUnaG, respectively. Displayed values: Mean \pm SD. (E,F) Correlation between FWHM of vimentin filament and the sampling coverage for (E) UnaG- and (F) eUnaG-labeled vimentin filaments, with corresponding distribution of FWHM (upper histograms); 153 and 222 filaments were analyzed for UnaG and eUnaG, respectively. Shaded error regions indicate SDs at each FWHM.

Virant et al., 2018). The sampling coverages for UnaG and eUnaG from cells with normal expression patterns were largely similar (Figures 4C,D). We also obtained a similar distribution of fiber widths and similar correlations of fiber FWHM to the sampling coverage of individual fibers for UnaG and eUnaG (Figures 4E,F). The fiber widths and sampling coverages of UnaG and eUnaG were on par with the pair of BC2 tag and nanobodies (Virant et al., 2018), indicating that both UnaG and eUnaG are incorporated into the vimentin fibrils at proper cytosolic concentrations. For both UnaG- and eUnaG-labeled fibrils, the sampling coverage increases as the width increases, approaching the complete sampling of fibrils exceeding a width of $200 \mathrm{~nm}$ (Figures 4E,F). However, the coverage for the thinnest fibers was slightly $(\sim 10 \%)$ higher for eUnaG-labeled fibrils. Also, for widths in 100-200 nm, the coverage of eUnaG-labeled fibrils was plateaued to $100 \%$, while the coverage of UnaGlabeled fibrils remained well below $100 \%$. These differences contributed to a slightly higher mean value and a narrower deviation in the distribution of sampling coverage for eUnaGlabeled fibrils (Figures 4C,D). In addition, the bright UnaG patches found in many UnaG-expressing cells indicate the tendency of UnaG to aggregate at high cytosolic concentration
(Figures 1F,G). Altogether, eUnaG provides a way for highquality SMLM imaging in green emission channel with better expression patterns and lower labeling artifacts.

\section{DISCUSSION}

We demonstrated the capacity of eUnaG as a potential fluorescent marker for SMLM in the green emission channel. Like UnaG, eUnaG can undergo repetitive fluorescence switching cycles required for SMLM, and the on- and off-switching are mediated by the rebinding and photo-oxidation of bilirubin, respectively. A single eUnaG molecule offers a slightly higher photon number than $\mathrm{UnaG}$, and thus, it can potentially provide the highest spatial resolution among the existing FPs for SMLM. In addition to the unique benefits in SMLM, the clear improvements in labeling artifact of eUnaG make it a much better probe for SMLM as well as conventional diffractionlimited imaging.

The slight increase in photon number (Figure 2) may appear inconsistent with the significant increase in bulk fluorescence intensity (Figures 1B,D). However, the population 
of UnaG with non-linear fluorescence intensity to the expression level (Figure 1C), the higher level of aggregation in OSER (Supplementary Figure 3), and the bright circular patches found in $49 \%$ of Vim-UnaG cells (Figures 1F,G and Supplementary Figure 5) support the hypothesis of aggregation-induced reduction of fluorescence. The similar lifetimes of UnaG and eUnaG (Supplementary Figure 2) rule out the possibility of aggregation-induced quenching, leaving aggregation-induced inhibition of ligand binding as a plausible cause. Restriction of access to ligands reduces the number of fluorescent UnaG molecules, but does not influence the single-molecule brightness.

Enhanced UnaG can easily label protein targets via standard genetic incorporation and transient expression in mammalian cells. eUnaG provides better expression patterns than UnaG, especially for proteins like vimentin whose structure can be easily perturbed by protein aggregation. Short peptide tags such as BC2 tag and ALFA tag have shown a similarly low level of labeling artifacts in vimentin fibers (Virant et al., 2018; Götzke et al., 2019). However, the fluorescent labeling of the tags requires delivery of fluorophore-conjugated nanobodies. For instance, anti-BC2 nanobody was delivered into live cells by protein transfection. In contrast, bilirubin, the small-molecule ligand, is cell-permeable and can be delivered into live and fixed cells by simply adding it to the culture medium (Kwon et al., 2020). Moreover, while the non-specifically bound fluorescent nanobodies can increase background fluorescence, the nonfluorescent bilirubin ligands only become fluorescent after binding to UnaG and exhibit negligible non-specific background fluorescence in conventional (Supplementary Figure 11) and SMLM imaging even under Epi-illumination (Kwon et al., 2020).

The lower labeling artifact of eUnaG may be attributed to the stability of the protein and the ligand. The thermal stability of eUnaG was higher than UnaG (Yeh et al., 2017). Also, the bilirubin ligand in eUnaG was more rigid than that of UnaG due to the stronger interaction network among the ligand, the protein, and more stably bound water molecules in the binding cavity that supplement more hydrogen bonds (Lee et al., 2018). The higher stability of eUnaG may help to decrease the aggregation tendency. This is reminiscent of our observation that $49 \%$ of Vim-UnaGexpressing cells contained a large patch of aggregated proteins (Figure 1F, "Patch"). These circular patches in the juxtanuclear regions have the characteristics of collapsed vimentin structures associated with aggresome (Supplementary Figure 12; Johnston et al., 1998). Aggresome formation indicates that overexpression of Vim-UnaG produced a large amount of misfolded proteins, making the cells to employ proteostasis processes (Kawaguchi et al., 2003). In contrast, such aggregated structure was not found in any Vim-eUnaG-expressing cell.

In fact, eUnaG fused to vimentin may become a valuable tool for investigating proteostasis. Since the discovery of vimentin's

\section{REFERENCES}

Betzig, E., Patterson, G. H., Sougrat, R., Lindwasser, O. W., Olenych, S., Bonifacino, J. S., et al. (2006). Imaging intracellular fluorescent proteins at nanometer resolution. Science 313, 1642-1645. doi: 10.1126/science.1127344

Brakemann, T., Stiel, A. C., Weber, G., Andresen, M., Testa, I., Grotjohann, T., et al. (2011). A reversibly photoswitchable GFP-like protein with fluorescence presence at the aggresome (Johnston et al., 1998), many studies have used vimentin as a marker for aggresome formation. Recent studies have begun to unveil the function of vimentin at the aggresome (Morrow and Moore, 2020). For example, vimentin recruits proteasome machineries to the aggresome, thereby playing a key role in stem cell proteostasis (Morrow et al., 2020). eUnaG may help to resolve ultrastructures and dynamics of vimentin at the aggresome in vivo, thereby revealing the organismal implications in aging and cancer.

We anticipate that eUnaG's suppressed labeling artifact and excellent SMLM capabilities would make it a new workhorse in live-cell SMLM imaging.

\section{DATA AVAILABILITY STATEMENT}

The raw data supporting the conclusions of this article will be made available by the authors, without undue reservation.

\section{AUTHOR CONTRIBUTIONS}

S-HS proposed and designed the idea of this work. SK prepared the biological samples, acquired and analyzed the confocal images, evidence of photoswitching, and kinetics information. JK acquired the widefield and SMLM images and analyzed the single-molecule characteristics and sampling coverage. All authors contributed to the writing and manuscript revision.

\section{FUNDING}

This work was supported by the Institute for Basic Science (IBSR023-D1).

\section{ACKNOWLEDGMENTS}

We thank Jong-Seok Park in the Ulsan National Institute of Science and Technology for generating the eUnaG-containing plasmids and his advisor, Prof. Hyun-Woo Rhee, in the Seoul National University for kindly providing the genetic constructs.

\section{SUPPLEMENTARY MATERIAL}

The Supplementary Material for this article can be found online at: https://www.frontiersin.org/articles/10.3389/fmolb. 2021.647590/full\#supplementary-material

excitation decoupled from switching. Nat. Biotechnol. 29, 942-947. doi: 10.1038/ nbt.1952

Chudakov, M. D., Matz, M. V., Lukyanov, S., and Lukyanov, K. A. (2010). Fluorescent proteins and their applications in imaging living cells and tissues. Physiol. Rev. 90, 1103-1163. doi: 10.1152/physrev.00038.2009

Costantini, L. M., Fossati, M., Francolini, M., and Snapp, E. L. (2012). Assessing the tendency of fluorescent proteins to oligomerize under 
physiologic conditions. Traffic 13, 643-649. doi: 10.1111/j.1600-0854.2012.01 336. $\mathrm{x}$

Dempsey, G. T., Vaughan, J. C., Chen, K. H., Bates, M., and Zhuang, X. (2011). Evaluation of fluorophores for optimal performance in localization-based super-resolution imaging. Nat. Methods 8, 1027-1036. doi: 10.1038/nmeth. 1768

Edelstein, A. D., Tsuchida, M. A., Amodaj, N., Pinkard, H., Vale, R. D., and Stuurman, N. (2014). Advanced methods of microscope control using $\mu$ Manager software. J. Biol. Methods 1:e10. doi: 10.14440/jbm. 2014.36

Endesfelder, U., Malkusch, S., Fricke, F., and Heilemann, M. (2014). A simple method to estimate the average localization precision of a single-molecule localization microscopy experiment. Histochem. Cell. Biol. 141, 629-638. doi: 10.1007/s00418-014-1192-1193

Götzke, H., Kilisch, M., Martínez-Carranza, M., Sograte-Idrissi, S., Rajavel, A., Schlichthaerle, T., et al. (2019). The ALFA-tag is a highly versatile tool for nanobody-based bioscience applications. Nat. Commun. 10, 4403-4412. doi: 10.1038/s41467-019-12301-12307

Hess, S. T., Girirajan, T. P. K., and Mason, M. D. (2006). Ultra-high resolution imaging by fluorescence photoactivation localization microscopy. Biophys. J. 91, 4258-4272. doi: 10.1529/biophysj.106.091116

Johnston, J. A., Ward, C. L., and Kopito, R. R. (1998). Aggresomes: a cellular response to misfolded proteins. J. Cell Biol. 143, 1883-1898. doi: 10.1083/jcb. 143.7.1883

Kaberniuk, A. A., Mohr, M. A., Verkhusha, V. V., and Snapp, E. L. (2018). moxMaple3: a photoswitchable fluorescent protein for PALM and protein highlighting in oxidizing cellular environments. Sci. Rep. 8:14738. doi: 10.1038/ s41598-018-32955-32955

Kawaguchi, Y., Kovacs, J. J., McLaurin, A., Vance, J. M., Ito, A., and Yao, T.P. (2003). The deacetylase HDAC6 regulates aggresome formation and cell viability in response to misfolded protein stress. Cell 115, 727-738. doi: 10.1016/ s0092-8674(03)00939-935

Kumagai, A., Ando, R., Miyatake, H., Greimel, P., Kobayashi, T., Hirabayashi, Y., et al. (2013). A bilirubin-inducible fluorescent protein from eel muscle. Cell 153, 1602-1611. doi: 10.1016/j.cell.2013.05.038

Kwon, J., Park, J.-S., Kang, M., Choi, S., Park, J., Kim, G. T., et al. (2020). Bright ligand-activatable fluorescent protein for high-quality multicolor livecell super-resolution microscopy. Nat. Commun. 11:273. doi: 10.1038/s41467019-14067-14064

Lee, E., Shim, S.-H., and Cho, M. (2018). Fluorescence enhancement of a ligandactivated fluorescent protein induced by collective noncovalent interactions. Chem. Sci. 9, 8325-8336. doi: 10.1039/c8sc03558j

Marsh, R. J., Pfisterer, K., Bennett, P., Hirvonen, L. M., Gautel, M., Jones, G. E., et al. (2018). Artifact-free high-density localization microscopy analysis. Nat. Methods 15, 689-692. doi: 10.1038/s41592-018-0072-75

McKinney, S. A., Murphy, C. S., Hazelwood, K. L., Davidson, M. W., and Looger, L. L. (2009). A bright and photostable photoconvertible fluorescent protein. Nat. Methods 6, 131-133. doi: 10.1038/nmeth. 1296

Morrow, C. S., and Moore, D. L. (2020). Vimentin's side gig: regulating cellular proteostasis in mammalian systems. Cytoskeleton 77, 515-523. doi: 10.1002/cm. 21645

Morrow, C. S., Porter, T. J., Xu, N., Arndt, Z. P., Ako-Asare, K., Heo, H. J., et al. (2020). Vimentin coordinates protein turnover at the aggresome during neural stem cell quiescence exit. Cell Stem Cell 26, 558-568.e9. doi: 10.1016/j.stem. 2020.01.018

Ovesný, M., Kř́ížek, P., Borkovec, J., Svindrych, Z., and Hagen, G. M. (2014). ThunderSTORM: a comprehensive ImageJ plug-in for PALM and STORM data analysis and super-resolution imaging. Bioinformatics 30, 2389-2390. doi: 10.1093/bioinformatics/btu202
Park, J.-S., Nam, E., Lee, H.-K., Lim, M. H., and Rhee, H.-W. (2016). In cellulo mapping of subcellular localized bilirubin. ACS Chem. Biol. 11, 2177-2185. doi: 10.1021/acschembio.6b00017

Patterson, G. H., and Lippincott-Schwartz, J. (2002). A photoactivatable GFP for selective photolabeling of proteins and cells. Science 297, 1873-1877. doi: 10. 1126/science. 1074952

Rodriguez, E. A., Campbell, R. E., Lin, J. Y., Lin, M. Z., Miyawaki, A., Palmer, A. E., et al. (2017). The growing and glowing toolbox of fluorescent and photoactive proteins. Trends Biochem. Sci. 42, 111-129. doi: 10.1016/j.tibs.2016.09.010

Rust, M. J., Bates, M., and Zhuang, X. (2006). Sub-diffraction-limit imaging by stochastic optical reconstruction microscopy (STORM). Nat. Methods 3, 793-796. doi: 10.1038/nmeth929

Sauer, M., and Heilemann, M. (2017). Single-molecule localization microscopy in eukaryotes. Chem. Rev. 117, 7478-7509. doi: 10.1021/acs.chemrev.6b00667

Sharonov, A., and Hochstrasser, R. M. (2006). Wide-field subdiffraction imaging by accumulated binding of diffusing probes. Proc. Natl. Acad. Sci. U S A. 103, 18911-18916. doi: 10.1073/pnas.0609643104

Shcherbakova, D. M., Sengupta, P., Lippincott-Schwartz, J., and Verkhusha, V. V. (2014). Photocontrollable fluorescent proteins for superresolution imaging. Annu. Rev. Biophys. 43, 303-329. doi: 10.1146/annurev-biophys-051013-22836

Shitashima, Y., Shimozawa, T., Kumagai, A., Miyawaki, A., and Asahi, T. (2017). Two distinct fluorescence states of the ligand-induced green fluorescent protein UnaG. Biophys. J. 113, 2805-2814. doi: 10.1016/j.bpj.2017.10.022

Shroff, H., Galbraith, C. G., Galbraith, J. A., and Betzig, E. (2008). Live-cell photoactivated localization microscopy of nanoscale adhesion dynamics. Nat. Methods 5, 417-423. doi: 10.1038/nmeth.1202

Subach, F. V., Patterson, G. H., Manley, S., Gillette, J. M., Lippincott-Schwartz, J., and Verkhusha, V. V. (2009). Photoactivatable mCherry for high-resolution two-color fluorescence microscopy. Nat. Methods 6, 153-159. doi: 10.1038/ nmeth.1298

Thompson, R. E., Larson, D. R., and Webb, W. W. (2002). Precise nanometer localization analysis for individual fluorescent probes. Biophys. J. 82, 27752783. doi: 10.1016/S0006-3495(02)75618-X

To, T. L., Zhang, Q., and Shu, X. (2016). Structure-guided design of a reversible fluorogenic reporter of protein-protein interactions. Protein Sci. 25, 748-753. doi: $10.1002 /$ pro. 2866

Virant, D., Traenkle, B., Maier, J., Kaiser, P. D., Bodenhöfer, M., Schmees, C., et al. (2018). A peptide tag-specific nanobody enables high-quality labeling for dSTORM imaging. Nat. Commun. 9:930. doi: 10.1038/s41467-018-03191-3192

Wang, S., Moffitt, J. R., Dempsey, G. T., Xie, X. S., and Zhuang, X. (2014). Characterization and development of photoactivatable fluorescent proteins for single-molecule-based superresolution imaging. Proc. Natl. Acad. Sci. U S A. 111, 8452-8457. doi: 10.1073/pnas.1406593111

Yeh, J. T.-H., Nam, K., Yeh, J. T.-H., and Perrimon, N. (2017). eUnaG: a new ligand-inducible fluorescent reporter to detect drug transporter activity in live cells. Sci. Rep. 7:41619. doi: 10.1038/srep41619

Zhou, X. X., and Lin, M. Z. (2013). Photoswitchable fluorescent proteins: ten years of colorful chemistry and exciting applications. Curr. Opin. Chem. Biol. 17, 682-690. doi: 10.1016/j.cbpa.2013.05.031

Conflict of Interest: The authors declare that the research was conducted in the absence of any commercial or financial relationships that could be construed as a potential conflict of interest.

Copyright (c) $2021 \mathrm{Ko}$, Kwon and Shim. This is an open-access article distributed under the terms of the Creative Commons Attribution License (CC BY). The use, distribution or reproduction in other forums is permitted, provided the original author(s) and the copyright owner(s) are credited and that the original publication in this journal is cited, in accordance with accepted academic practice. No use, distribution or reproduction is permitted which does not comply with these terms. 